\title{
Low-dimensional spike rate dynamics of coupled adaptive model neurons
}

\author{
Moritz Augustin ${ }^{1,2^{*}}$, Josef Ladenbauer ${ }^{1,2}$, Klaus Obermayer ${ }^{1,2}$ \\ From 24th Annual Computational Neuroscience Meeting: CNS*2015 \\ Prague, Czech Republic. 18-23 July 2015
}

The spiking activity of single neurons can be well described by a two-dimensional integrate-and-fire model that includes neuronal adaptation [1] caused by slowly decaying potassium currents [2]. For fluctuating inputs sparsely coupled spiking model neurons exhibit stochastic population dynamics which can be effectively characterized using the Fokker-Planck equation (see, e.g., [3-5]). This approach leads to a model with an infinitedimensional state space and non-standard boundary conditions. However, the spike rate dynamics can be approximated by a low-dimensional ordinary differential equation in different ways $[4,6,7]$. Although these approximation techniques are interrelated it is not clear which reduced model best reproduces the spike rate of the original spiking network, depending on the statistics of the input. Here we first extend each of these reduction methods to account for neuronal adaptation and then evaluate the reduced models in terms of spike rate reproduction accuracy for a range of biologically plausible input statistics, computational demand and implementation complexity

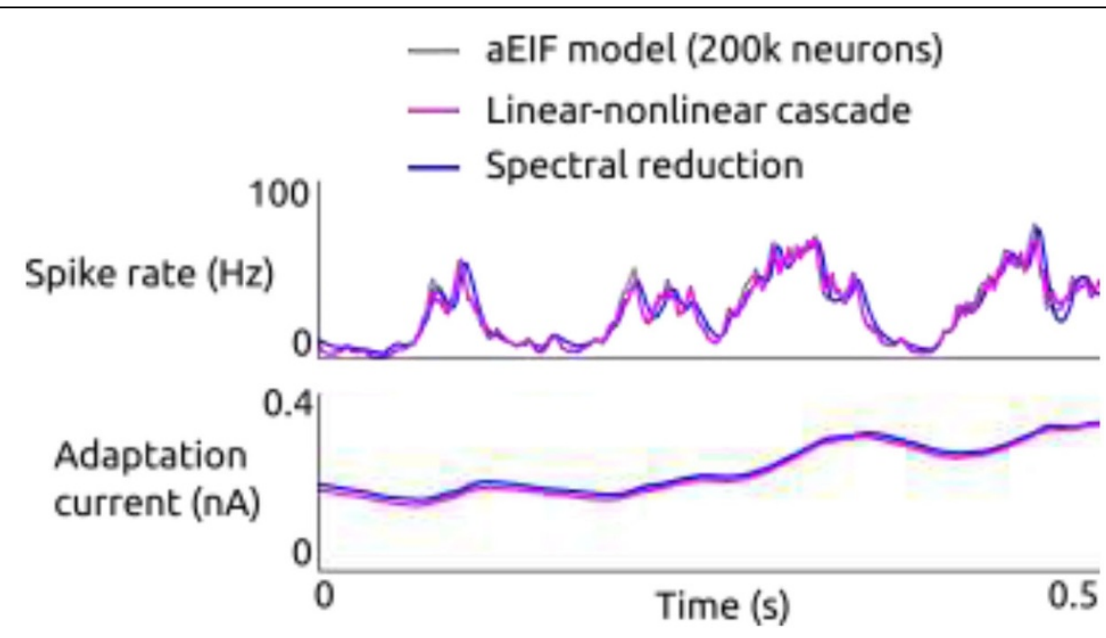

Figure 1 Simulation of a large population of adaptive exponential integrate-and-fire (aEIF) neurons driven by a stochastic current with time-varying moments. Instantaneous spike rate and adaptation current averaged over 200,000 neurons are shown in gray. Overlaid are mean spike rate and adaptation current of two derived low-dimensional models receiving input with the same time-dependent moments as the population of aEIF neurons.

\footnotetext{
* Correspondence: augustin@ni.tu-berlin.de

'Neural Information Processing Group, Berlin Institute of Technology, Berlin,

Germany

Full list of author information is available at the end of the article
}

(c) 2015 Augustin et al. This is an Open Access article distributed under the terms of the Creative Commons Attribution License (http:// creativecommons.org/licenses/by/4.0), which permits unrestricted use, distribution, and reproduction in any medium, provided the original work is properly cited. The Creative Commons Public Domain Dedication waiver (http://creativecommons.org/publicdomain/ zero/1.0/) applies to the data made available in this article, unless otherwise stated. 
(see, e.g., Figure 1). These reduced descriptions are well suited for (i) application in neural mass/mean-field based brain network models, having a link to single neuron properties retained and being computationally efficient, and (ii) mathematical analyses of, e.g., asynchronous and rhythmic network states.

\section{Acknowledgements}

This work was supported by the DFG Collaborative Research Center SFB910.

\section{Authors' details}

${ }^{1}$ Neural Information Processing Group, Berlin Institute of Technology, Berlin, Germany. ${ }^{2}$ Bernstein Center for Computational Neuroscience Berlin, Berlin, Germany.

Published: 18 December 2015

\section{References}

1. Brette R, Gerstner W: Adaptive exponential integrate-and-fire model as an effective description of neuronal activity. J Neurophysiol 2005,

94:3637-3642.

2. Ladenbauer J, Augustin M, Obermayer K: How adaptation currents change threshold, gain, and variability of neuronal spiking. J Neurophysiol 2014, 111:939-953.

3. Brunel N: Dynamics of sparsely connected networks of excitatory and inhibitory spiking neurons. J Comput Neurosci 2000, 8:183-208.

4. Mattia M, Del Giudice P: Population dynamics of interacting spiking neurons. Phys Rev E 2002, 66:051917.

5. Augustin $M$, Ladenbauer J, Obermayer K: How adaptation shapes spike rate oscillations in recurrent neuronal networks. Front Comput Neurosci 2013, 7:9.

6. Schaffer E, Ostojic S, Abbott L: A complex-valued firing-rate model that approximates the dynamics of spiking networks. PLOS Comput Biol 2013 9:e1003301.

7. Ostojic S, Brunel N: From spiking neuron models to linear-nonlinear models. PLOS Comput Biol 2011, 7:e1001056.

doi:10.1186/1471-2202-16-S1-P183

Cite this article as: Augustin et al.: Low-dimensional spike rate dynamics of coupled adaptive model neurons. BMC Neuroscience 2015 16(Suppl 1): P183.

\section{Submit your next manuscript to BioMed Central and take full advantage of:}

- Convenient online submission

- Thorough peer review

- No space constraints or color figure charges

- Immediate publication on acceptance

- Inclusion in PubMed, CAS, Scopus and Google Scholar

- Research which is freely available for redistribution

Submit your manuscript at www.biomedcentral.com/submit
Biomed Central 